$\$$ Research Square

\title{
Repurposing FDA-approved drugs against the "main protease" pivotal enzyme in COVID-19 virus using computer-aided drug design techniques
}

\author{
Mahmoud Al-Sha'er ( $\sim$ a.mahmoud@zu.edu.jo) \\ Zarqa University https://orcid.org/0000-0001-9962-1971 \\ Qosay Al Balas \\ Jordan University of Science and Technology
}

\section{Research Article}

Keywords: COVID19, Antiviral, Structure Based Design, Remdisivir, Lopinavir, Pharmacophore Modeling

Posted Date: November 8th, 2021

DOI: https://doi.org/10.21203/rs.3.rs-1056609/v1

License: @) (1) This work is licensed under a Creative Commons Attribution 4.0 International License. Read Full License 


\section{Abstract}

Background: Severe acute respiratory syndrome coronavirus 2 (SARS-CoV-2) is considered an unprecedented global pandemic in the modern era, infecting millions of people with an approximately $2 \%$ death rate. It emerged in late 2019 and researchers worldwide race time to identify druggable targets where medicinal chemists can design potential inhibitors.

Objective: Drug repurposing is one of the most convenient methods available now to find already approved drugs for human use with a potential tendency to cure COVID-19.

Method: Within this work, the FDA-approved drug database containing 1281 drugs was employed to extract possible drugs that could have activity against the "main protease" enzyme in this virus. Various computer-aided drug design techniques, such as pharmacophore generation, ligand pharmacophore mapping, molecular docking and filtering techniques, were used to identify potential inhibitors. The cocrystallized ligand inside the main protease enzyme was utilized to generate two structure-based pharmacophores that were used as templates for deletion in the FDA database. To validate the selection of the active hits from pharmacophore mapping, molecular docking via "LibDock" was performed, while the highest scoring candidate was selected.

Results: Interestingly, three antiviral drugs (lopinavir, saquinavir and remdesivir) were qualified to be potential main protease inhibitors.

Conclusion: The researchers in this work recommend clinically investigating the possibility of these drugs as preliminary therapies until selective drugs for COVID-19 virus are approved.

\section{Introduction}

Severe acute respiratory syndrome coronavirus 2 (SARS-CoV-2) is the originator of the COVID-19 pandemic and is accused of causing congeries of contagions with approximately two million casualities worldwide (1). This disease is the third outbreak of coronavirus strains in this century, which is preceded by 2003 SARS and 2012 MERS (Middle East respiratory syndrome) (2, 3). It originated in China and huge efforts were spent to prevent its spread; unfortunately, these efforts failed and were classified as a pandemic (4). Considerable efforts have been attempted to discover selective potent small molecules with anti-MERS-CoV activity (5). Potential candidates were recognized via screening FDA-approved medicines and drug-like small molecules using cell-based experimentation and in vitro scanning (6). A series of available computer-aided drug design (CADD) techniques are available to assist in discovering potential drugs, such as structure-based drug design, ligand-based drug design and Denovo drug design (7). The main protease enzyme of SARS-CoV-2 is an auspicious target for designing an effective drug, as this enzyme is pivotal for the viability and replication of the coronavirus $(4,8)$. Luckily, the crystal structure of the COVID-19 protease enzyme cocrystallized with inhibitors has been deposited in the Protein Data Bank (9). This crystal structure is a very important starting point for structure-based drug design to find selective lead compounds that are able to inhibit COVID-19 (10).

In this work, a rational methodology based on CADD techniques was employed to repurpose the currently available FDAapproved drugs for the treatment of COVID-19, which has the advantage of saving time and rescuing infected people by giving them possible inhibitors of the main protease enzyme. Structure-based and ligand-based techniques were used in this research in this work by subjecting the FDA approved drug to different selection criteria aiming to reach drugs with high affinity to the enzyme $(11,12)$.

\section{Materials And Methods Molecular modeling Ligand preparation}


The FDA database with 1281 drugs was downloaded from the "Zinc Database" (13), and the ligands were prepared for molecular docking by exploiting the "Prepare ligands" protocol supported by Discovery Studio 2019 from Biovia Inc. (San Diego, CA, www.biovia.com) (14). Within this protocol, the drug molecules will be formulated in a reasonable starting point by giving proper ionization, generating tautomers, generating isomers, fixing bad valences and assigning 3D coordinates. Structure drawing was performed by employing Chem Draw 12.0 (15).

\section{Protein preparation}

The 3D coordinates of the COVID-19 main protease enzyme PDB code 6lu7 were obtained from PDB (16). The "Prepare protein" protocol was employed to make the enzyme ready for docking and structure-based drug design. This protocol performs Gasteiger-Marsili charge assignment, standardizes atom names, inserts missing atoms in residues and removes alternate conformations, inserts missing loop regions based on either SEQRES data, optimizes short- and medium-sized loop regions with the LOOPER algorithm and calculates the pKa and protonates the structure. Integral water molecules were retained, and the main protease was used without energy minimization.

\section{Receptor-ligand pharmacophore generation}

The "interaction pharmacophore generation" protocol uses the nonbond interactions between the "input receptor" and the "input ligand" to enumerate pharmacophores. The pharmacophores were ranked with a GFA (genetic function approximation) model, and the top pharmacophores containing minimum features to maximum features were reported. The employed parameters were specified as follows: "minimum number of features" was 4 while the "maximum number of features" was 10 , the "number of generated pharmacophoric models" was 10 and the cocrystallized water was retained in the protein structure. The Receptor-Ligand Pharmacophore Generation protocol was employed to consummate this task with preset parameters. This protocol engenders discriminatory pharmacophore models placed on receptor-ligand interactions. First, a group of features from the co-crystallized ligand were identified. The following predefined feature types are considered: hydrogen bond acceptor (HBA), hydrogen bond donor (HBD), hydrophobic (Hbic), negative ionizable (NI), positive ionizable (PI) and ring aromatic (RA) (CATALYST 4.11 Users' Manual) (17). Second, the pharmacophore models were graded based on an estimate of selectivity score, and then the ten highest models were returned.

\section{Pharmacophore mapping}

This protocol maps and aligns ligands to a generated pharmacophore. Catalysts were used to pinpoint ligands that matched a pharmacophore, and the ligands were allineated to the query. It expresses the results with a fitting score where the higher values indicated better fitting.

The screened drugs (1281 FDA-approved drugs) were mapped on the two generated pharmacophore models, Hypo(6LU7_2_01) and Hypo(6LU7_2_02), using the "best fit" option in Biovia 2019, followed by summation of the fit values. Then, the captured drugs were sorted and ranked according to the sum of fit values. Finally, the revealed mapped drugs were docked into the binding site of COVID-19 using rigid-body docking parameters.

\section{Molecular docking}

The three dimensional coordinates of the COVID-19 PDB code 6lu7 were retrieved from PDB $(9,16)$, and the Gasteiger-Marsili charges were designed to the protein atoms. Integral $\mathrm{H}_{2} \mathrm{O}$ molecules were maintained, and the protein was utilized with no energy minimization. Docking criteria: LibDock is a site-feature docking algorithm that berth ligands into an active site navigated by binding hotspots. This docking setting contemplates the docility of the ligand while considering the receptor adamant (18).

The following LibDock specifications were applied in this project: (i) Prior to docking, the Discovery Studio module CATCONFIRM was used to engender 255 conformers at max (not violating an energy brink of $20 \mathrm{kcal} / \mathrm{mol}$ from the most stable conformer) for each ligand utilizing fast conformation generation preference. (ii) An active site sphere of $13.85 \AA$ radius enclosing the midpoint of the cocrystallized ligand was set. (iii) The maximum number of binding site hotspots was set to 
100. (iv) Ligand-to-hotspots matching the RMSD tolerance value were set to $0.25 \AA$. (v) The maximum number of poses saved for every ligand within hotspot mapping before final posture minimization $=100$. (vi) Maximum number of postires to be kept for every ligand in the binding vicinity $=100$. (vii) Minimum LibDock grade (poses below this value are not retrieved) $=100$. (viii) Maximum number of rigid body minimization steps during the final pose optimization (using the BFGS method) $=50$. (ix) The maximum number of steric clashes granted before the pose-hotspot alignment is aborted (specified as a fraction of the heavy atom count) $=0.1$. (x) Maximum value for nonpolar solvent reachable surface area for a particular pose to be recoded as successful $=15.0 \AA ̊ 2$. (xi) Maximum value for polar solvent reachable solvent area for a specific posture to be reported as successful = $5.0 \AA ̊ 2$. (xii) No final ligand minimization was employed (i.e., in the binding pocket), and the volume of the binding site equaled $64.19 \AA^{3}(19-22)$.

\section{Results \& Discussion}

\section{Structure-based pharmacophore generation}

The objective of this research is to scrutinize anti-main protease drug candidates as potential COVID-19 inhibitors from already subsisting drugs as a repurposing technique. This is achieved in this work by using a well-established drug discovery scenario named "structure-based pharmacophore". The main protease enzyme with its cocrystallized ligand has been delved to unravel its pharmacophoric entities. Two pharmacophoric models were produced, as shown in Tables 1 and 2 and Figures 1 and 2.

Table 1

Pharmacophore generated from the co-crystallized COVID-19 structure (6LU7)

\begin{tabular}{|lllll|}
\hline Pharmacophore & Model & Number of Features & Feature Set* & Selectivity Score \\
\hline Hypo(6LU7_2_01) & Pharmacophore_01 & 4 & ADDD & 8.76 \\
\hline Hypo(6LU7_2_02) & Pharmacophore_02 & 4 & ADDH & 7.85 \\
\hline *A: Hydrogen bond acceptor, D: Hydrogen bond donor, H: Hydrophobe & \\
\hline
\end{tabular}


Table 2. Pharmacophoric features and corresponding weights, tolerances and 3D coordinates of generated pharmacophore from co-crystallized COVID-19

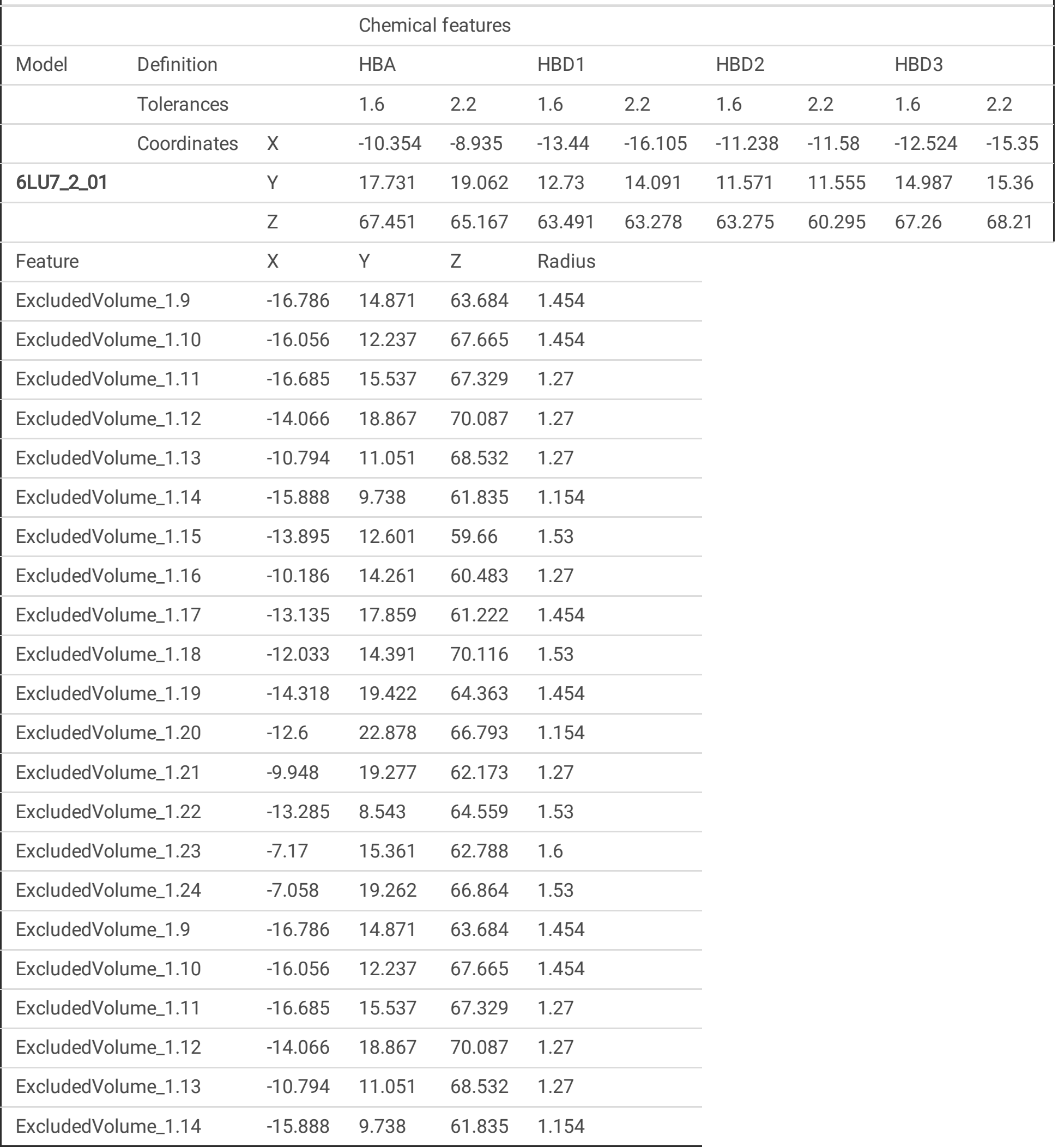


Continue Table 2. Pharmacophoric features and corresponding weights, tolerances and 3D coordinates of generated pharmacophore from co-crystallized COVID-19

\begin{tabular}{|c|c|c|c|c|c|c|c|c|c|}
\hline \multirow{3}{*}{$\begin{array}{l}\text { Model } \\
\text { 6LU7_2_02 }\end{array}$} & \multirow[b]{2}{*}{ Definition } & & \multicolumn{6}{|c|}{ Chemical features } & \multirow[b]{2}{*}{$\mathrm{HBIC}$} \\
\hline & & & \multicolumn{2}{|l|}{ HBA } & \multicolumn{2}{|l|}{ HBD1 } & \multicolumn{2}{|l|}{ HBD2 } & \\
\hline & \multicolumn{2}{|l|}{ Tolerances } & 1.6 & 2.2 & 1.6 & 2.2 & 1.6 & 2.2 & 1.6 \\
\hline & \multirow[t]{3}{*}{ Coordinates } & $x$ & -10.354 & -8.93514 & -13.44 & -16.105 & -12.524 & -15.3462 & -10.7 \\
\hline & & $\mathrm{Y}$ & 17.731 & 19.0619 & 12.73 & 14.091 & 14.987 & 15.361 & 12.38 \\
\hline & & Z & 67.451 & 65.1672 & 63.491 & 63.278 & 67.26 & 68.2061 & 64.24 \\
\hline \multicolumn{2}{|l|}{ Feature } & $x$ & $\mathrm{Y}$ & Z & Radius & & & & \\
\hline \multicolumn{2}{|c|}{ ExcludedVolume_1.8 } & -16.786 & 14.871 & 63.684 & 1.454 & & & & \\
\hline \multicolumn{2}{|c|}{ ExcludedVolume_1.9 } & -16.056 & 12.237 & 67.665 & 1.454 & & & & \\
\hline \multicolumn{2}{|c|}{ ExcludedVolume_1.10 } & -16.685 & 15.537 & 67.329 & 1.27 & & & & \\
\hline \multicolumn{2}{|c|}{ ExcludedVolume_1.11 } & -14.066 & 18.867 & 70.087 & 1.27 & & & & \\
\hline \multicolumn{2}{|c|}{ ExcludedVolume_1.12 } & -10.794 & 11.051 & 68.532 & 1.27 & & & & \\
\hline \multicolumn{2}{|c|}{ ExcludedVolume_1.13 } & -15.888 & 9.738 & 61.835 & 1.154 & & & & \\
\hline \multicolumn{2}{|c|}{ ExcludedVolume_1.14 } & -13.895 & 12.601 & 59.66 & 1.53 & & & & \\
\hline \multicolumn{2}{|c|}{ ExcludedVolume_1.15 } & -10.186 & 14.261 & 60.483 & 1.27 & & & & \\
\hline \multicolumn{2}{|c|}{ ExcludedVolume_1.16 } & -13.135 & 17.859 & 61.222 & 1.454 & & & & \\
\hline \multicolumn{2}{|c|}{ ExcludedVolume_1.17 } & -12.033 & 14.391 & 70.116 & 1.53 & & & & \\
\hline \multicolumn{2}{|c|}{ ExcludedVolume_1.18 } & -14.318 & 19.422 & 64.363 & 1.454 & & & & \\
\hline \multicolumn{2}{|c|}{ ExcludedVolume_1.19 } & -12.6 & 22.878 & 66.793 & 1.154 & & & & \\
\hline \multicolumn{2}{|c|}{ ExcludedVolume_1.20 } & -9.948 & 19.277 & 62.173 & 1.27 & & & & \\
\hline \multicolumn{2}{|c|}{ ExcludedVolume_1.21 } & -13.285 & 8.543 & 64.559 & 1.53 & & & & \\
\hline \multicolumn{2}{|c|}{ ExcludedVolume_1.22 } & -7.17 & 15.361 & 62.788 & 1.6 & & & & \\
\hline \multicolumn{2}{|c|}{ ExcludedVolume_1.23 } & -7.058 & 19.262 & 66.864 & 1.53 & & & & \\
\hline \multicolumn{2}{|c|}{ ExcludedVolume_1.8 } & -16.786 & 14.871 & 63.684 & 1.454 & & & & \\
\hline \multicolumn{2}{|c|}{ ExcludedVolume_1.9 } & -16.056 & 12.237 & 67.665 & 1.454 & & & & \\
\hline \multicolumn{2}{|c|}{ ExcludedVolume_1.10 } & -16.685 & 15.537 & 67.329 & 1.27 & & & & \\
\hline \multicolumn{2}{|c|}{ ExcludedVolume_1.11 } & -14.066 & 18.867 & 70.087 & 1.27 & & & & \\
\hline ExcludedVol & Ime_1.12 & -10.794 & 11.051 & 68.532 & 1.27 & & & & \\
\hline ExcludedVol & me_1.13 & -15.888 & 9.738 & 61.835 & 1.154 & & & & \\
\hline
\end{tabular}

\section{Pharmacophore mapping}

The "Ligand Profiler" tool was utilized to scrutinize the capability of the engendered pharmacophores to retrieve active main protease inhibitors from a library of 1281 FDA drugs, as illustrated in Table $3(11,12)$. Lopinavir scored the highest fit value for the sum of the two pharmacophores (6.84), in which it scored the highest on Hypo(6LU7_2_02) with a fitting value of 3.66. 
Saquinavir had the next highest total score of 6.78 and ranked first in mapping Hypo(6LU7_2_01). Remdesivir was third in queue, with a total score of 6.77 , which was slightly different from saquinavir. However, it has proximal fitting values for both pharmacophores, which is the best in satisfying the two generated pharmacophores.

Figure 2A shows the interaction of the cocrystallized ligand (6LU7) with the amino acids in the binding pocket, namely, Glu166, Gln189, Gly143, His163, His164, Phe140, Met165, Thr25 and Thr190. These interactions are declared by three hydrogen bond donor (HBD), hydrogen bond acceptor (HBA), hydrophobic ( $\mathrm{Hbic}$ ) features in addition to exclusion spheres that reflect the unfavorable interaction represented in two models: Hypo(6LU7_2_01) and Hypo(6LU7_2_02). By assessing the mapping features of Hypo(6LU7_2_01) with the cocrystallized ligand (N3) displayed in Figures 2A and 2C, the pyrrole NH functionality is mapped with the carbonyls of Glu166 and Phe140. The OH substituent on the pyrrole ring acts as an HBD towards His163. The adjacent NH of the amide complements the carbonyl group of Gln189. Moreover, the corresponding carbonyl functionality correlates to the NH backbone of Glu166 and Met165.

\begin{tabular}{|c|c|c|c|c|}
\hline & \multicolumn{4}{|c|}{ Table 3: Antiviral drugs mapped with COVID-19 co-crystallized pharmacophore model } \\
\hline & Drug Name & Hypo(6LU7_2_01) & Нypo(6LU7_2_02) & Sum of fit value \\
\hline 1 & Lopinavir & 3.17424 & 3.66905 & 6.84 \\
\hline 2 & Saquinavir & 3.69998 & 3.0818 & 6.78 \\
\hline 3 & Remdesivir & 3.50298 & 3.26887 & 6.77 \\
\hline 4 & Raltegravir & 3.42645 & 3.25023 & 6.68 \\
\hline 5 & Indinavir & 3.33961 & 3.15649 & 6.50 \\
\hline 6 & Ritonavir & 3.4135 & 2.69569 & 6.11 \\
\hline 7 & Entecavir & 2.96574 & 2.89141 & 5.86 \\
\hline 8 & Nelfinavir & 2.04368 & 3.48115 & 5.52 \\
\hline 9 & Darunavir & 1.7563 & 3.03795 & 4.79 \\
\hline 10 & Abacavir & 2.19382 & 2.26266 & 4.46 \\
\hline 11 & Amprenavir & 0.88646 & 3.17231 & 4.06 \\
\hline 12 & Alithiomycin & 0.38969 & 3.24623 & 3.62 \\
\hline 13 & Valacyclovir & 2.28102 & 1.06015 & 3.34 \\
\hline 14 & Penciclovir & 3.33872 & 0 & 3.34 \\
\hline 15 & Ganciclovir & 3.33321 & 0 & 3.33 \\
\hline 16 & Ribavirin & 3.13763 & 0 & 3.14 \\
\hline 17 & Cidofovir & 2.74535 & 0 & 2.75 \\
\hline 18 & Favirapivir & 0 & 2.71624 & 2.72 \\
\hline 19 & Zanamivir & 2.21 & 0 & 2.21 \\
\hline 20 & Atazanavir & 0 & 2.10294 & 2.1 \\
\hline 21 & Oseltamivir & 0 & 1.89534 & 1.90 \\
\hline
\end{tabular}

The hypo(6LU7_2_02) mapping pattern is portrayed in Figures 2A and 2D. It is epitomized by forming Vander wall interactions between the oxazole ring and the hydrophobic fraction of amino acids Met165, Phe140, and His163. Moreover, the carbonyl of the amide is mapped with the NH group of Glu166 and His164 via hydrogen bonding, while the hydroxyl of the pyrrole ring 
matches His 163 with hydrogen bonds. The adjacent NH group maps GIn189 via hydrogen bond. It is worth mentioning here that the mapping of the N3 cocrystallized inhibitor with both Hypo(6LU7_2_01) and Hypo(6LU7_2_02) described in Figure 2 is in agreement with the original binding pattern of N3, an enlightenment that our work is valid and that the generated results are authentic.

Figure 3 shows the mapped poses of compounds 1, 2 and 3 (lopinavir, saquinavir and remdesivir). This pattern of binding for lopinavir is shown in Figures 3A and 3B for Hypo(6LU7_2_01) and Hypo(6LU7_2_02), respectively, and is summarized in Table 4.

Table 4

Binding pattern of lopinavir with Hypo(6LU7_2_01) and Hypo(6LU7_2_02).

\begin{tabular}{|c|c|c|c|}
\hline \multicolumn{4}{|c|}{ Lopinavir Interactions } \\
\hline \multicolumn{2}{|c|}{ Hypo(6LU7_2_01) } & \multicolumn{2}{|c|}{ Hypo(6LU7_2_02) } \\
\hline HBD & Amide $\mathrm{NH}$ next to xylene ring & HBD & Amide $\mathrm{NH}$ next to xylene ring \\
\hline HBD & Secondary alcohol & HBD & Amide $\mathrm{NH}$ adjacent to tetrahydropyridinone \\
\hline HBD & Amide $\mathrm{NH}$ adjacent to tetrahydropyridinone & HBA & Amide carbonyl adjacent to tetrahydropyridinone \\
\hline HBA & Carbonyl group of the tetrahydropyridinone & Hbic & Phenyl ring \\
\hline
\end{tabular}

Figures 3C, 3D show how saquinavir mapped with the two pharmacopheric models in a similar way as lopinavir. Figures 3E and 3F show how remdesivir mapped with the (6LU7_2_01) and Hypo(6LU7_2_02) models in which part of the aminopyrrolo triazine ring matches the Hbic feature, whereas the phosphoryl amino group maps HBA, while the two hydroxyl groups in the tetrahydrofuran ring map the two HBD features. Finally, the NH group of the phosphoryl amino group maps the HBD feature in the second pharmacophore (Table 5).

Table 5

Binding pattern of remdesivir with Hypo(6LU7_2_01) and Hypo(6LU7_2_02).

\begin{tabular}{|llll|}
\hline \multicolumn{2}{|l|}{ Remdesivir Interactions } & \\
\hline Hypo(6LU7_2_01) & Hypo(6LU7_2_02) \\
\hline HBD & Hydroxyl of THF ring & HBD & Hydroxyl of THF ring \\
\hline HBD & 2nd Hydroxyl of THF ring & HBD & 2nd Hydroxyl of THF ring \\
\hline HBD & NH group of the phosphoryl amino & HBA & Carbonyl of the ester group \\
\hline HBA & Carbonyl of the ester group & Hbic & Part of pyrrolo triazine ring \\
\hline
\end{tabular}

\section{Molecular docking}

LibDock is integrated and validated docking software in Discovery 2019 that was used to dock the highest fitting drugs resulting from pharmacophore mapping. Interestingly, the three drugs with the highest scores were remdesivir, saquinavir and lopinavir, with ranking scores ranging from 115.3 for remdesivir to 108.8 for lopinavir (Table 6). The results obtained from molecular docking and pharmacophore mapping have affirmed the concept the researchers build their work as there is a noticeable similarity of the main protease enzyme in many viruses such as BatCoV RaTG13 pro and with the previous SARS$\operatorname{CoV}(4,23,24)$. 
Table 6

LibDock of the highest fitting antiviral drugs ; Lopinavir, Saquinavir and Remdesivir into the binding pocket of COVID-19 main protease enzyme*

\begin{tabular}{|llll|}
\hline Drug Name & Number of generated conformers & Number of docked poses & LibDock score of the best pose \\
\hline LOPINAVIR & 248 & 66 & 108.8 \\
\hline SAQUINAVIR & 69 & 23 & 113.8 \\
\hline REMDESIVIR & 254 & 93 & 115.3 \\
\hline *(pdb :6LU7, resolution $2.16 \AA)$ www.rcsb.org & & \\
\hline
\end{tabular}

From the current results, it can be recommended that the current antiviral drugs are good candidates to treat COVID-19 until future vaccines or new more potent inhibitors come to the market with more selectivity and safety profiles. On the other hand, other drugs from different classes have shown reasonable inhibitory activity against the COVID-19 main protease that deserves to be forwarded for clinical trials to investigate their effectiveness. The advantageous facet in this process is that there is no need to enter the lengthy cycle of drug approval and clinical trials.

\section{Discovery route}

Within this section, the sequence of steps performed in this project starting from the crystal structure of the main protease enzyme from COVID-19 ending with the recommended drugs that deserve to be investigated to tackle the truculent behavior of this virus is shown in Figure 4.

The work started by generating structure-based pharmacophore models of the active site of the main protease enzyme benefiting from a cocrystallized ligand. The interactions were sketched and evaluated while the corresponding pharmacophores were generated and successfully managed to obtain two pharmacophores. In the next step, a database of 1281 FDA-approved drugs was screened on the generated pharmacophores with the successful retrieval of 154 drugs. SMART and Lipiniski filters were employed to shorten the list, and 99 hit drugs succeeded in passing the filters. Finally, docking results approved 21 candidate drugs as potential inhibitors, with lopinavir, saquinavir and remdesivir being the most promising ones, as shown in Figure 5.

\section{Conclusion}

Two structure-based pharmacophore models were generated with the aid of the co-crystallized ligand inside the active site of the COVID-19 "main protease". Pharmacophore mapping of FDA-approved drugs over the generated pharmacophore has retrieved a series of drugs that could be candidates for main protease inhibition. A confirmatory step was performed by docking the retrieved drugs to evaluate their effectiveness in active site binding. This work is an emergency and necessary step for repurposing currently used medicines for the treatment of COVID-19 until the conventional pipeline of drug discovery begets its active drugs.

\section{Declarations}

\section{ETHICS APPROVAL AND CONSENT TO PARTICIPATE}

Not applicable

\section{HUMAN AND ANIMAL RIGHTS}

No humans or animals were used for studies that are the basis of this research.

\section{CONSENT FOR PUBLICATION}

Not applicable. 


\section{AVAILABILITY OF DATA AND MATERIALS}

Not applicable.

\section{FUNDING}

Zarqa University and Jordan University of Science and Technology

\section{CONFLICT OF INTEREST}

The authors declare no conflicts of interest, financial or otherwise.

\section{ACKNOWLEDGEMENTS}

We thank the Deanship of Scientific Research at Zarqa University for their generous funds and the Deanship of Scientific Research at Jordan University of Science \& Technology; project No. 128/2020.

\section{SUPPLEMENTARY MATERIAL}

Supplementary material is available on the publisher's website along with the published article.

\section{References}

(1) E. Dong, H. Du, L. Gardner. An interactive web-based dashboard to track COVID-19 in real time. Lancet Infect Dis. 20, (2020) 533-534; doi: 10.1016/S1473-3099(20)30120-1.

(2) Y. Yang, F. Peng, R. Wang, K. Guan, T. Jiang, G. Xu, J. Sun, C. Chang. The deadly coronaviruses: The 2003 SARS pandemic and the 2020 novel coronavirus epidemic in China. J. Autoimmun. 109 (2020) 102434; doi: 10.1016/j.jaut.2020.102434.

(3) V.G. da Costa, M.L. Moreli, M.V. Saivish, The emergence of SARS, MERS and novel SARS-2 coronaviruses in the 21st century. Arch Virol. 165 (2020) 1517-1526; https://doi.org/10.1007/s00705-020-04628-0.

(4) S. Ullrich, C. Nitsche. The SARS-CoV-2 main protease as drug target, Bioorg. Med. Chem. Let. 30 (2020) 127377; doi: https://doi.org/10.1016/j.bmcl.2020.127377.

(5) A.H. de Wilde, D. Jochmans, C. C. Posthuma, J. C. Zevenhoven-Dobbe, S. van Nieuwkoop, T. M. Bestebroer, B. G. van den Hoogen, J. Neyts, E. J. Snijder, Screening of an FDA-approved compound library identifies four small-molecule inhibitors of Middle East respiratory syndrome coronavirus replication in cell culture, Antimicrob. Agents Chemother. 58 (2014) 48754884. doi: 10.1128/AAC.03011-14.

(6) M.A. Farha, E.D. Brown, Drug repurposing for antimicrobial discovery. Nat. Microbiol. 4 (2019) 565-577 ; https://doi.org/10.1038/s41564-019-0357-1.

(7) G. Sliwoski, S. Kothiwale, J. Meiler, E. W. Lowe, L. B. Jr Eric, Computational methods in drug discovery, Pharmacol. Rev. 66 (2014) 334-395. doi: 10.1124/pr.112.007336.

(8) R. Yoshino, N. Yasuo, M. Sekijima, Identification of key interactions between SARS-CoV-2 main protease and inhibitor drug candidates. Scientific Reports 10 (2020) 12493; https://doi.org/10.1038/s41598-020-69337-9.

(9) The crystal structure of COVID-19 main protease in complex with an inhibitor N3: http://www.rcsb.org/structure/6LU7.

(10) G. Gilliland, H.M. Berman, H. Weissig, I.N. Shindyalov, J. Westbrook, P.E. Bourne, et al. The Protein Data Bank. Nucleic Acids Research 28 (2000) 235-42; https://doi.org/10.1093/nar/28.1.235. 
(11) M.A. Al-Sha'er, I. Mansi, M. Khanfar, A.Abudayyh, Discovery of new heat shock protein 90 inhibitors using virtual cocrystallized pharmacophore generation. J. Enzyme Inhib. Med. Chem. 31 (2016) 64-77;

https://doi.org/10.1080/14756366.2016.1218485.

(12) M.A. Al-Sha'er, I. Mansi, I. Almazari, N. Hakooz, Evaluation of novel Akt1 inhibitors as anticancer agents using virtual cocrystallized pharmacophore generation. J. Mol. Graph. Model. 62 (2015) 213-25; doi: 10.1016/j.jmgm.2015.10.004. Epub 2015 Oct.

(13) J.J. Irwin, B.K. Shoichet, ZINC-a free database of commercially available compounds for virtual screening. J. Chem. Inf. Model., 45 (2005) 177-182; https://doi.org/10.1021/ci049714+.

(14) Dassault Systèmes BIOVIA, Discovery Studio Modeling Environment, Release 2017, San Diego: Dassault Systèmes, 2016.

(15) Cambridge Soft Corp., Cambridge, MA, http://www.cambridgesoft.Com, 2020.

(16) Protein Data Bank, 2015 (PDB : http://www.rcsb.org)

(17) Catalyst 4.10, (2000). LigandFit User Manual; Biovia software Inc.: San Diego, CA.

(18) L. Pinzi, G. Rastelli, Molecular Docking: Shifting Paradigms in Drug Discovery. Int. J. Mol. Sci. 20 (2019) 4331; DOI: 10.3390/ijms20184331.

(19) M.A. Al-Sha'er, Q.A. Al-Balas, M.A. Hassan, G.A. Al Jabal, A.M. Almaaytah, Combination of Pharmacophore Modeling and 3D-QSAR analysis of Potential Glyoxalase-I Inhibitors as Anticancer Agents. Comput. Biol Chem. 80 (2019) 102-110; doi: 10.1016/j.compbiolchem.

2019.03.011.

(20) M.A. Al-Sha'er, R.A. Al-Aqtash, M.O. Taha, Discovery of New Phosphoinositide 3-kinase Delta (PI3Kס) Inhibitors via Virtual Screening using Crystallography-derived Pharmacophore Modelling and QSAR Analysis. Med Chem. 15 (2019) 588-601; DOI: $10.2174 /$

1573406415666190222125333

(21) I.A. Mansi, M.A. Al-Sha'er, N.M. Mhaidat, M.O. Taha, R. Shahin, Investigation of binding characteristics of Phosphoinositide-dependent kinase-1 (PDK1) co-crystallized ligands through virtual pharmacophore modeling leading to novel anti - PDK1 hits, Med. Chem. 2020;16(7):860-880. doi: 10.2174/1573406415666190724131048.

(22) M.A. Al-Sha'er, Q.A. Al-Balas, M.A. Hassan, G.A. Al Jabal, A.M. Almaaytah, Combination of pharmacophore modeling and 3D-QSAR analysis of potential glyoxalase-I inhibitors as anticancer agents, Comput. Biol. Chem. 80 (2019) 102-110; doi: 10.1016/j.compbiolchem.2019.03.011.

(23) M. Stoermer, Homology Models of Coronavirus 2019-nCoV 3CLpro Protease. ChemRxiv (2020) DOI: 10.26434/chemrxiv.11637294.v3.

(24) C. Wu, Y. Liu, Y. Yang, P. Zhang, W. Zhong, Y. Wang, Q. Wang, Y. Xu, M. Li, X. Li, M. Zheng, L. Chen, H. Li, Analysis of therapeutic targets for SARS-CoV-2 and discovery of potential drugs by computational methods. Acta Pharm. Sin. B. 10 (2020) 766-788; doi: 10.1016/j.apsb.2020.02.008. Epub 2020 Feb 27.

\section{Figures}




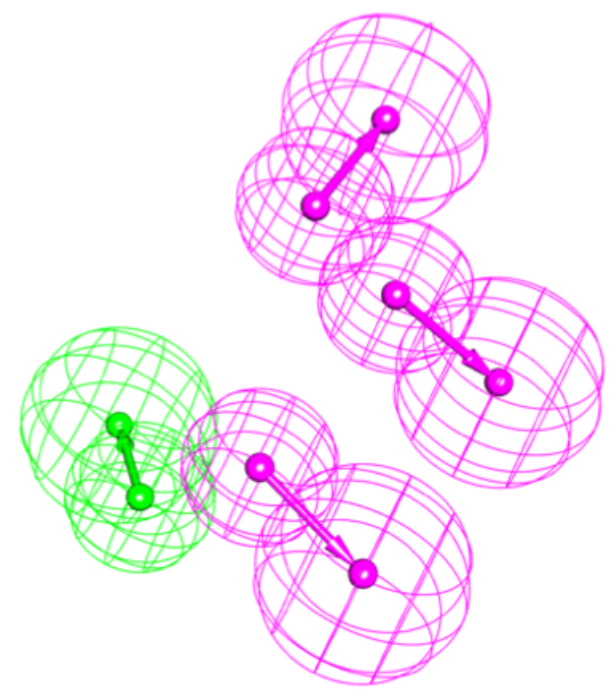

(A)

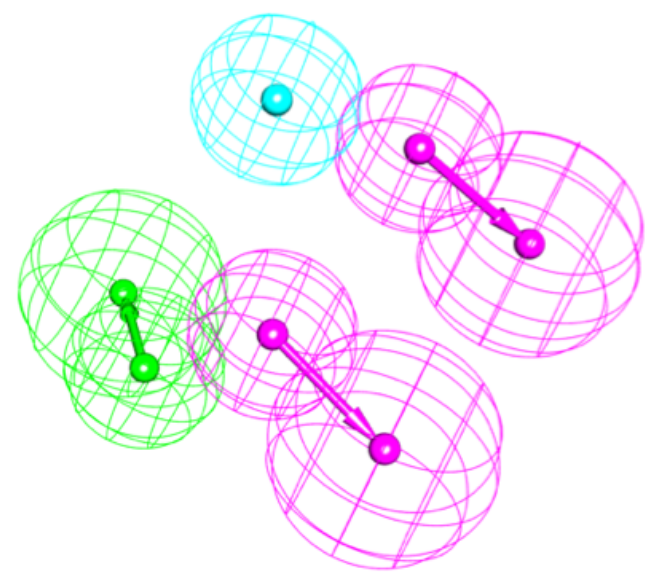

(C)

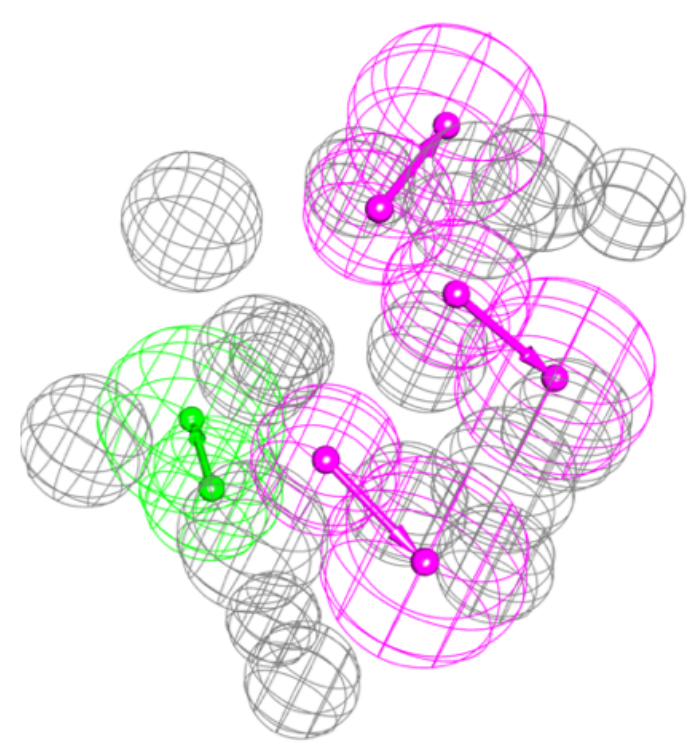

(B)

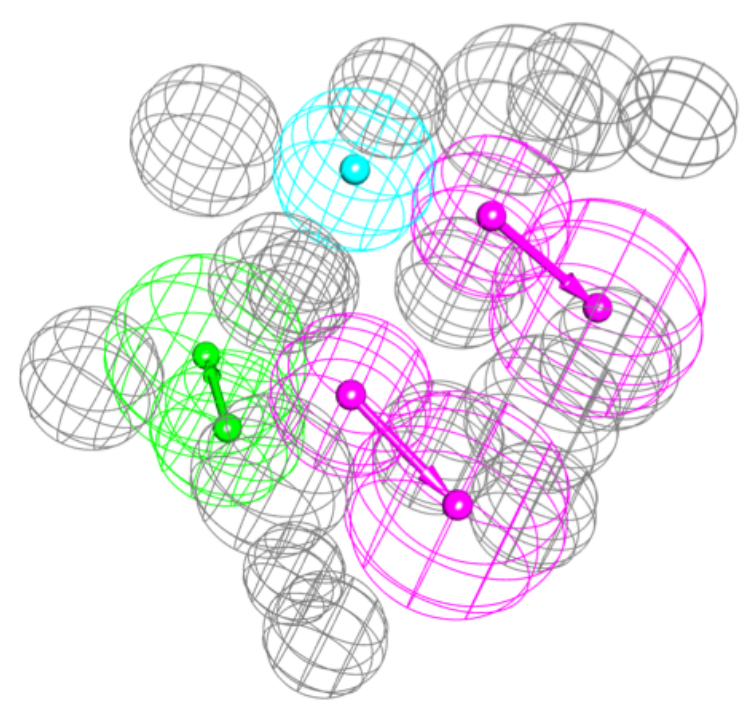

(D)

\section{Figure 1}

(A) unrefined pharmacophore model Hypo(6LU7_2_01) without exclusion volumes, (B) refined pharmacophore model Hypo (6LU7_2_01) with exclusion volumes, (C) unrefined pharmacophore model Hypo(6LU7_2_02) without exclusion volumes, (D) refined pharmacophore model Hypo (6LU7_2_02) with exclusion volumes, Hydrogen bond acceptor features (HBA) displayed as green vectored spheres, hydrogen bond donor feature (HBD) displayed as purple vectored spheres, aromatic ring feature (RingArom) displayed as orange vectored spheres, hydrophobic feature ( $\mathrm{Hbic}$ ) displayed as blue sphere. 

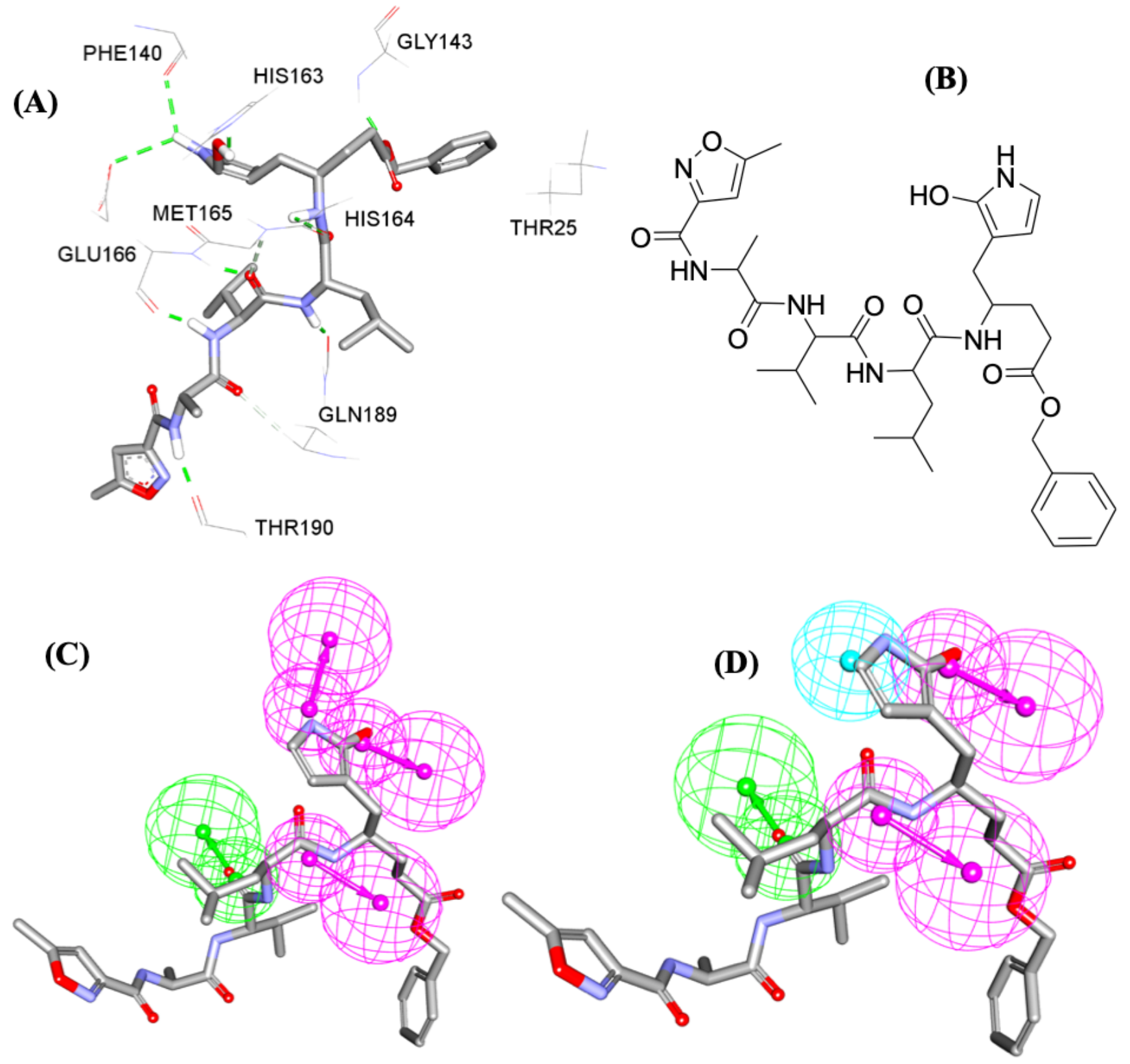

Figure 2

Binding interactions and analogous pharmacophoric features extracted from COVID-19 crystallographic complex 6LU7 (resolution $2.10 \AA$ A). (A) Binding interactions fastening the complexed ligand within COVID-19 (PDB 6LU7), (B) Chemical structure of cocrystallized ligand an inhibitor N3 (C) Hypo(6LU7_2_01) mapped with cocrystallized ligand an inhibitor N3. (D) Hypo(6LU7_2_02) mapped with the cocrystallized ligand inhibitor N3. 
(A)

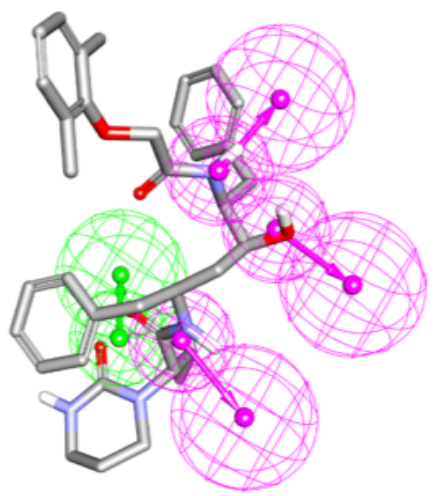

(C)

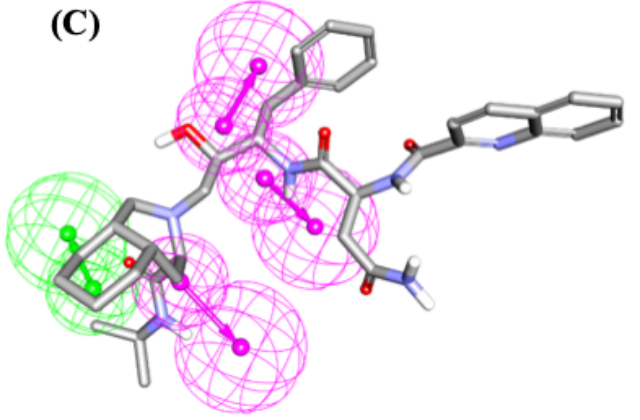

(E)

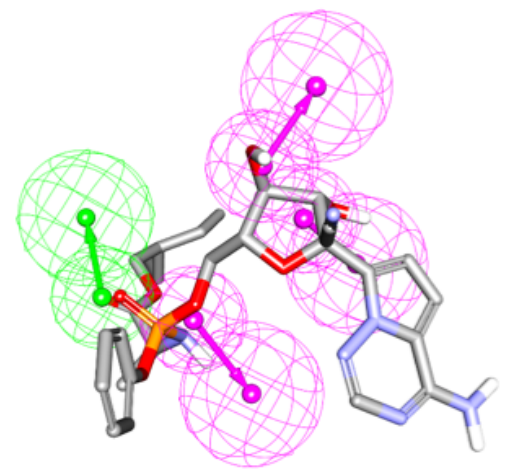

(B)

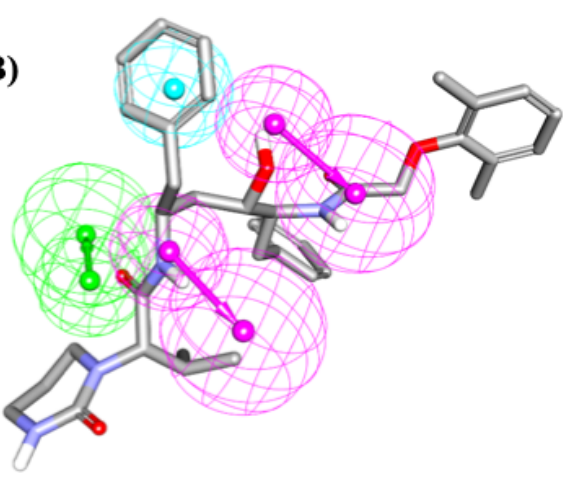

(D)

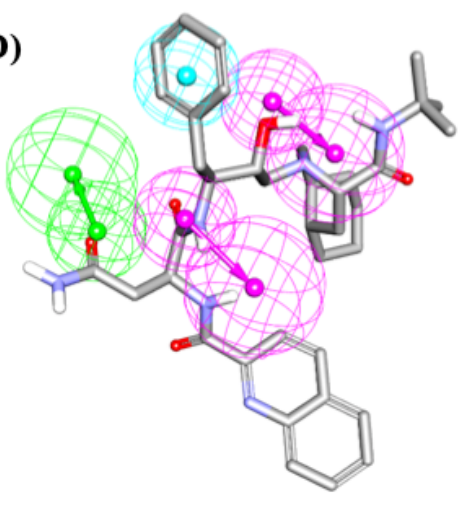

(F)

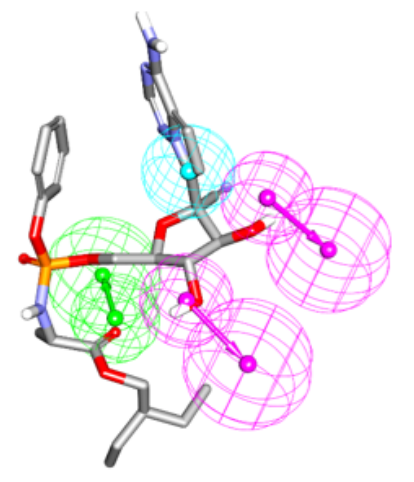

\section{Figure 3}

(A, C, E) Hypo(6LU7_2_01) mapped with Lopinavir, Saquinavir and Remdesivir respectively. (B, D, F) Hypo(6LU7_2_02) mapped with Lopinavir, Saquinavir and Remdesivir respectively. 
6LU7 COVID-19 cocrystallized protein

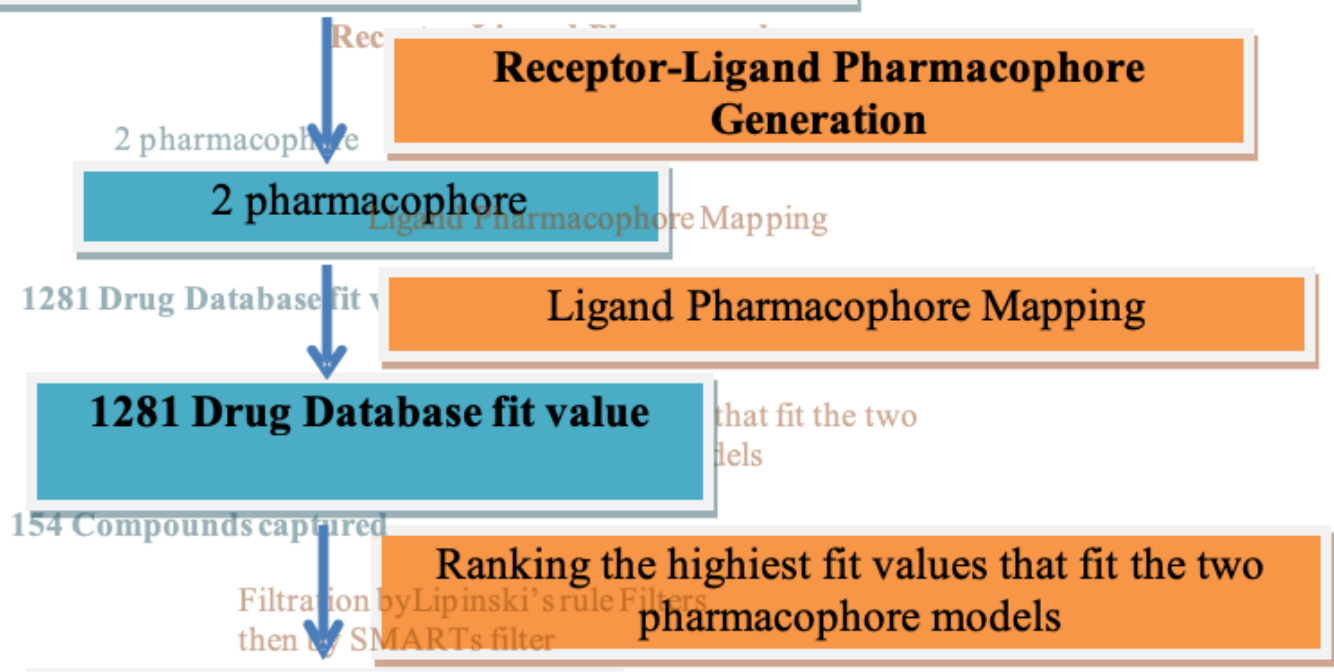

\section{Compounds captured}
P'Filtration byLipinski's rule Filters then by SMARTs filter

\section{Hits}

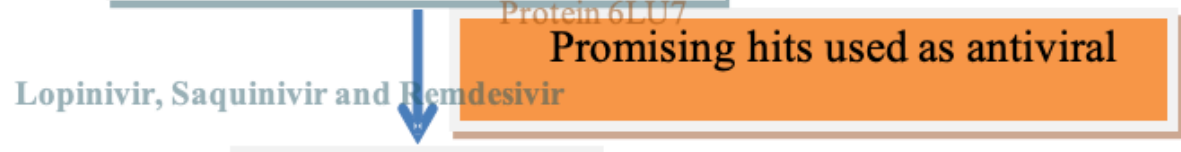

\section{Hits}

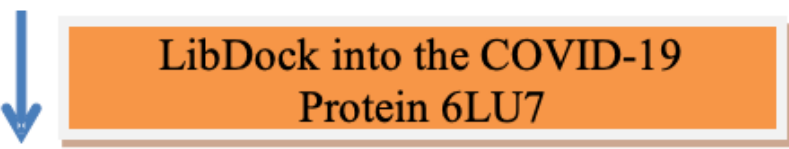

\section{Lopinivir, Saquinivir and Remdesivir}

\section{Figure 4}

The workflow pursued for the discovery of COVID-19 inhibitors started by screening the structural database of the drug list against two models: Hypo(6LU7_2_01) and Hypo(6LU7_2_02) 


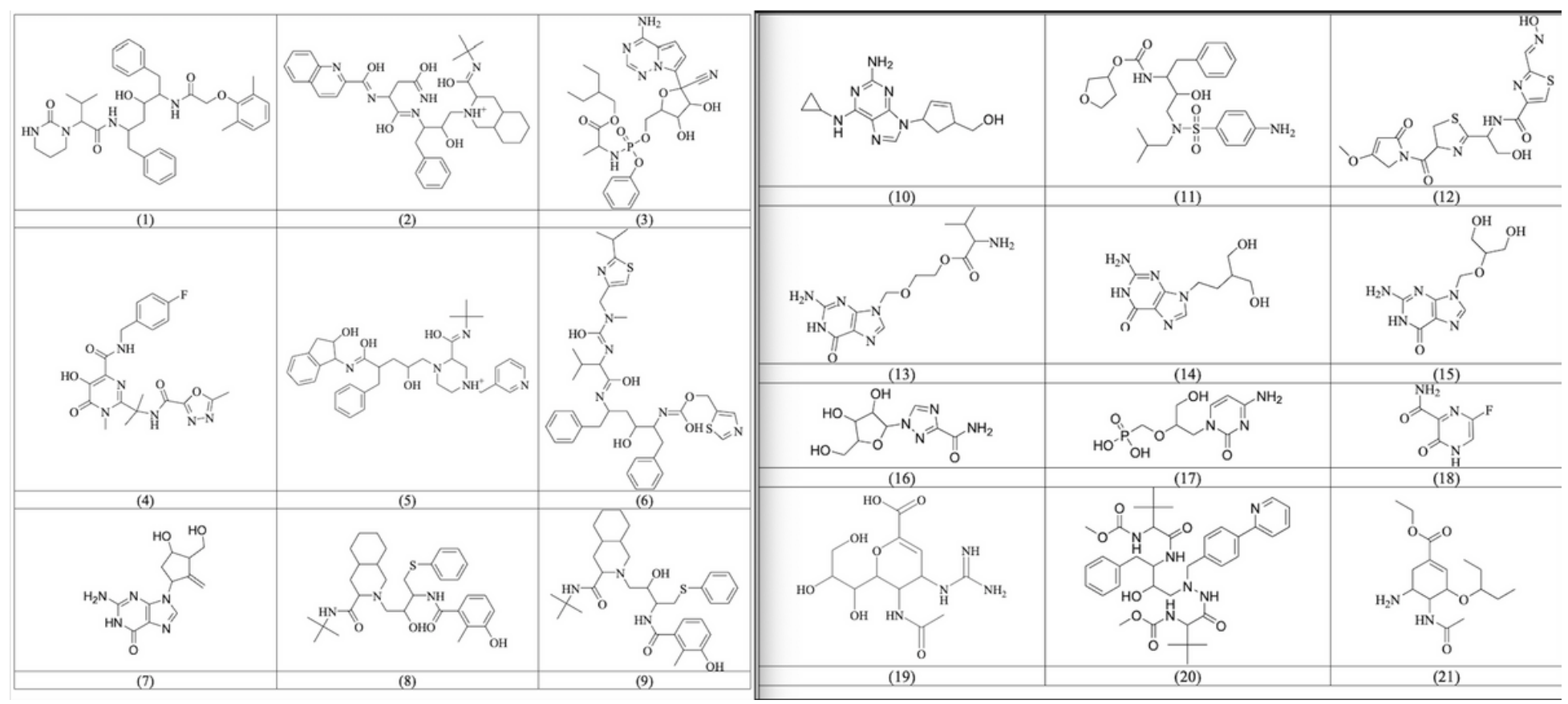

Figure 5

Chemical structure of In Silico high ranking captured drugs as Anti-COVID-19

\section{Supplementary Files}

This is a list of supplementary files associated with this preprint. Click to download.

- COVID19SuppMaterials2021.docx 